\title{
L'atoll polynésien de Mataiva face aux enjeux du phosphate
}

\section{Alexandre K. Magnan}

\section{(2) OpenEdition \\ 1 Journals}

\section{Édition électronique}

URL : http://journals.openedition.org/jso/8084

DOI : $10.4000 /$ jso.8084

ISSN : 1760-7256

\section{Éditeur}

Société des océanistes

\section{Édition imprimée}

Date de publication : 15 juillet 2018

Pagination : 259-264

ISBN : 978-2-85430-135-9

ISSN : 0300-953x

\section{Référence électronique}

Alexandre K. Magnan, «L'atoll polynésien de Mataiva face aux enjeux du phosphate », Journal de la Société des Océanistes [En ligne], 146 | 2018, mis en ligne le 15 juillet 2020, consulté le 25 juillet 2020. URL : http://journals.openedition.org/jso/8084 ; DOI : https://doi.org/10.4000/jso.8084 


\title{
Latoll polynésien de Mataiva face aux enjeux du phosphate
}

par

\author{
Alexandre K. MAGNAN*
}

\section{RÉSUMÉ}

L'atoll de Mataiva (Tuamotu, Polynésie française) possède un lagon "réticulé ", véritable curiosité géologique à la surface de la planète. Ce patrimoine naturel remarquable est cependant menacé, depuis les années 1970, par un projet d'exploitation du phosphate sur toute la moitié ouest du lagon, à l'initiative de diverses compagnies internationales et du gouvernement de Polynésie française, basé à Tahiti. Dans les années 1980, puis dans les années 2000, des rencontres ont été organisées avec les habitants de Mataiva, mais ces derniers se sont systématiquement opposés à ce projet, pour trois raisons principales: un attachement profond à cet atoll qu'il considèrent comme leur " mère nourricière "; le souvenir de n'avoir plus pu manger de poisson du lagon pendant 10 ans après la phase de test (qui n'a pourtant duré qu'un an et demi); et la peur de voir les zones d'exploitation s'étendre aux terres émergées. Si le projet n'a pas abouti jusqu'à maintenant, il n'a pas pour autant été abandonné.

Mots-CLÉs : Mataiva, phosphate, patrimoine géologique mondial

Cet article d'opinion met en lumière la menace qui pèse sur le lagon "réticulé " de l'atoll de Mataiva (Tuamotu, Polynésie française). Riche en phosphate, ce lagon est convoité depuis les années 1970 par des acteurs internationaux de l'extraction minière et par le gouvernement de Polynésie française, lequel y voit une source de revenus considérables. Or, exploiter le phosphate de Mataiva signifie creuser son lagon, et

\begin{abstract}
Mataiva Atoll (French Polynesia) has a $25 \mathrm{~km}^{2}$ entirely reticulated lagoon, a quite unique coral structure in the world. Mataiva lagoon is also rich in phosphate. The project of exploiting it arose in the 1970s, and in the 1980s international companies engaged a dialogue with the Government of French Polynesia to get authorisations. Public meetings were organized, but the inhabitants strongly opposed to the project, based on three main arguments related to (i) their respect towards the atoll, that they considered as "a mother providing survival means"; (ii) the negative effects on fishing they experienced after the 1.5-year testing extraction phase; (iii) and their conviction that companies would also try to extract phosphate from the islands themselves. This led to the abandonment of the project. A new one also failed in 2008 for the same reason. In January 2015, the Economic Council of French Polynesia however released a report partly dealing with the economic benefits to expect from atolls' phosphate, putting this geological patrimony once again at risk.
\end{abstract}

KEYwords: Mataiva, phosphate, geological world heritage

donc détruire de manière irréversible cette forme géologique quasi unique à l'échelle du globe. Si deux projets d'exploitation ont vu le jour depuis le début des années 1980, l'opposition de la population locale les a jusqu'à maintenant fait avorter. Mais de nouvelles velléités ont émergé depuis début 2015, qui posent une nouvelle fois la question du devenir de cette curiosité naturelle mondiale. Ce cas renvoie plus large-

* Institut du développement durable et des relations internationales, LIENSs, UMR 7266 CNRs/Université de La Rochelle. alexandre.magnan@iddri.org 
ment à la question récurrente des choix qu'il convient d'opérer, parfois, entre environnement et développement économique, et plus particulièrement ici, entre d'une part la préservation d'une richesse intrinsèque et d'un mode de vie relativement adapté aux contraintes environnementales, et d'autre part l'exploitation des ressources et les dommages irréversibles causés à l'environnement. C'est une histoire à l'image de nombreuses autres, qui voient depuis plusieurs siècles l'Humanité accumuler les erreurs, de la disparition des peuples indigènes à la destruction des forêts primaires, en passant aujourd'hui par la fonte des calottes polaires, mais sans pour autant en tirer assez d'enseignements pour inverser certaines tendances. Une prise de conscience est toutefois encore possible, et le cas de Mataiva peut y contribuer.

Ce texte fait suite à une mission de recherche effectuée sur l'atoll de Mataiva en février 2015, et dont le but initial était à la fois de caractériser le bâti et de dresser un premier bilan des impacts d'un cyclone, Niko, passé au plus près de l'île une dizaine de jours auparavant. Ce travail reposait en partie sur des entretiens (une vingtaine) auprès de la population locale. Les discussions que les entretiens généraient déviaient souvent sur le thème plus large des enjeux environnementaux et de développement de l'atoll. La question de l'exploitation du phosphate du lagon a ainsi rapidement émergé, si bien qu'au terme de la mission, $\mathrm{j}$ 'avais pu discuter longuement de cela avec cinq personnes, et mener deux entretiens poussés avec le maire en place, d'une part, et avec la Présidente de l'association de Mataiva contre le phosphate, d'autre part. J'ai également eu l'occasion, de m'entretenir longuement sur ce sujet avec un ancien maire de Mataiva, résidant aujourd'hui sur l'atoll voisin de Tikehau.

\section{Mataiva et son lagon réticulé}

À $300 \mathrm{~km}$ au nord de Tahiti, l'atoll de Mataiva $\left(14^{\circ} 55^{\prime} \mathrm{S}\right.$ et $\left.148^{\circ} 36^{\prime} \mathrm{W}\right)$ constitue la terminaison nord-ouest de la longue chaîne des Tuamotu-Gambier, en Polynésie française (Pacifique central). Celle-ci compte des atolls parmi les plus grands de la planète, tels ceux de Rangiroa $\left(1525 \mathrm{~km}^{2}\right)$ et de Fakarava $\left(1137 \mathrm{~km}^{2}\right)$, par exemple ${ }^{1}$. Celui de Mataiva est bien plus petit : $41 \mathrm{~km}^{2}$ de surface totale, dont seulement $16 \mathrm{~km}^{2}$ de terres émergées. Comme tous les atolls, Mataiva résulte de l'enfoncement progressif de volcans sous-marins ayant percé la surface de l'océan, puis ayant subi les assauts de l'érosion naturelle. Au fur et à mesure que les terres émergées s'enfonçaient, les récifs coralliens s'élargissaient pour devenir, au fil du temps, des anneaux plus ou moins grands, réguliers et continus : les atolls. Puis les vagues, notamment cycloniques, ont progressivement prélevé du matériau sur ces édifices coralliens pour l'accumuler en des endroits spécifiques de l'atoll. Ces tas de débris coralliens et de sable ont ensuite été naturellement colonisés par la végétation et diverses espèces animales, dont de nombreux oiseaux marins. Ainsi sont nées les îles coralliennes, que les habitants du Pacifique appellent les motu. Bien plus tard, les grandes pirogues se sont lancées dans un vaste voyage d'Ouest en Est à travers le Pacifique, avec à bord hommes, femmes, enfants, plantes et animaux domestiqués. Elles auraient atteint la Polynésie orientale il y a environ 2500 ans. S'il est difficile de savoir à quand remonte précisément le peuplement de l'atoll de Mataiva, il est sûr qu'il comptait seulement 126 habitants au début des années 1950 (Sodter, 1985), et 280 en 2012.

Aujourd'hui, les habitants de Mataiva vivent encore principalement de l'exploitation des cocoteraies pour la fabrication du coprah, qu'ils exportent ensuite vers Tahiti $^{2}$, et de la pêche, quasi exclusivement vivrière. La pêche se pratique à la fois dans le lagon et dans l'océan, et les poissons du lagon de Mataiva sont réputés pour être les plus goûteux des Tuamotu. Ils sont essentiellement capturés aux abords de la passe, côté océan comme côté lagon, et généralement à l'aide de pièges à poissons faits de bâtons de bois et de filets ${ }^{3}$. Mais la grande particularité de Mataiva, c'est son lagon "réticulé ", véritable curiosité géologique à la surface de la planète.

Le nom "Mataiva » signifie en tahitien "neufs yeux ", lesquels symbolisent les neuf hoa que compte l'atoll. Hoa est le nom tahitien qui désigne les chenaux qui, généralement envahis par la mer à marée haute, séparent les îles d'une même couronne récifale les unes des autres. Ces chenaux different de la "passe ", laquelle constitue un canal naturel en général assez profond (de quelques mètres à Mataiva à environ 50 mètres à Rangiroa, par exemple) et qui n'a jamais été colonisé par les récifs coralliens. La passe ouvre un passage entre les eaux du lagon et celles de l'océan, ce qui permet le renouvellement et

1. Rangiroa et Fakarava sont également des atolls des Tuamotu. Ils comptent respectivement 79 et $16 \mathrm{~km}^{2}$ de surface émergée correspondant aux îles basses qu'ils portent.

2. Activité économique peu rentable du fait de la concurrence mondiale et de l'arrivée sur le marché de produits de synthèse, la production de coprah est subventionnée aujourd'hui par le gouvernement de Polynésie française. Cela permet aux coprahiculteurs d'obtenir -120-140 Francs Pacifique (un peu plus de $1 €$ ) pour chaque kilo de coprah, et au gouvernement de maintenir une population sur les atolls et ainsi de limiter les migrations vers Tahiti (APF, 2017).

3. Le principe est simple : les poissons évoluant toujours dans le sens du courant, ils sont pris dans le piège qui forme une sorte d'entonnoir, incapables de rebrousser chemin. 
l'oxygénation des eaux du lagon. Vus d'avion, les hoa se différencient bien des passes : quand celles-ci affichent un bleu profond, les hoa virent aux turquoises et couleurs pastels, dévoilant ainsi leurs faibles profondeurs et leurs tapis de sable et de matériaux coralliens. Les hoa contribuent également au renouvellement des eaux du lagon, mais dans une bien moindre mesure par rapport à la passe. Ce n'est pourtant pas ce contexte morphologique peu favorable aux grands échanges océan-lagon qui explique la forme réticulée du lagon, laquelle a en réalité des origines bien plus anciennes (de l'ordre probablement de plusieurs millions d'années - Rougerie, 1995) et plus complexes (Delesalle et al., 1985 ; Rougerie et Jehl, 1994). Elles ont quoi qu'il en soit donné naissance à un lagon caractérisé par 70 "bassins " de 5 à $10 \mathrm{~m}$ de profondeur, chacun étant séparé des autres par de petits édifices coralliens, sortes de crêtes de montagnes sous-marines lilliputiennes. C'est une configuration très originale puisqu'un lagon se caractérise en général par un seul bassin de plusieurs dizaines de mètres de profondeur (60 m pour celui de Fakarava, par exemple). La littérature scientifique est très peu développée sur ces formes réticulées de lagon, et si quelquesunes ont été repérées dans certaines portions de lagons, il semblerait que Mataiva soit le seul lagon du monde à être entièrement réticulé. Cela en fait, parmi les centaines d'atolls que compte l'océan global, une exception géologique.

Le fait que la légende locale ait vu en les hoa les yeux de l'atoll, lequel est considéré par les habitants comme un être vivant à part entière, illustre le lien fort qui a existé, et qui existe encore, entre ces derniers et l'espace tant terrestre que marin.

Pour les habitants de Mataiva, l'atoll est :

" comme une maman, parce que c'est lui qui te donne à manger : le poisson et la noix de coco. Ici, sans argent, tu peux quand même vivre. L'atoll, c'est notre mère nourricière, et c'est pour ça qu'il faut en prendre soin et être reconnaissant envers la nature. » (Entretien du 12.02.2015 avec une femme âgée du village de Pahua, le seul de l'atoll)

Une telle appréhension de l'espace s'accompagne nécessairement d'un respect pour l'atoll en tant qu'entité, un respect pour la "nature", comme disent les habitants lorsqu'ils s'expriment en français. Et c'est ce respect profond qui les a amenés, depuis plus de vingt ans, à s'insurger contre le projet d'exploitation massive du phosphate dans le lagon. Mais l'histoire est loin d'être terminée, et ce patrimoine géologique rarissime est aujourd'hui toujours menacé de disparition, purement et simplement (Magnan et Duvat, 2015).

\section{Du phosphate et des hommes}

L'exploitation du phosphate est l'une des rares industries qui se sont développées dans les îles du Pacifique à partir du XIX ${ }^{e}$ siècle (Dupont et al., 1989 ; Fiollet, 2010 ; Pollock, 2014), notamment Nauru, Makatea en Polynésie française, et Banaba dans l'archipel de Kiribati. Ces trois îles ont hérité de leur longue fréquentation par de vastes colonies d'oiseaux marins la présence d'immenses gisements de phosphate ${ }^{6}$. Cet engrais naturel a été l'un des premiers à être utilisé pour engraisser les sols agricoles dans le Pacifique, que ce soit par des îles-continents comme l'Australie ou des îles montagneuses comme la NouvelleCalédonie. Dans des îles coralliennes pauvres en ressources naturelles, le phosphate est apparu comme une manne financière intéressante à exploiter car offrant des opportunités de développement. C'est dans les années 1970 que l'idée d'exploiter le phosphate du lagon de Mataiva ${ }^{7}$ a émergé, dans un contexte dans lequel l'exploitation du gisement de sa voisine Makatea prenait fin (1908-1966) (Decoudras et al., 2005).

Il a cependant fallu attendre le début des années 1980 pour que soient lancées les premières explorations visant à évaluer la qualité du phosphate de Mataiva. Ces tests, semble-t-il conduits par une société néo-calédonienne en partenariat avec une société australienne ${ }^{8}$, ont révélé la présence en abondance d'un phosphate de bien meilleure qualité que celui de Nauru (Lacour, 1986). Quantitativement, les estimations portaient le gisement à près de 15 millions de tonnes sur une surface couvrant la moitié occidentale du lagon, jusqu'à une profondeur de plusieurs dizaines de mètres. Le projet n'avait pas moins pour ambition que d'exploiter l'ensemble de ce gisement. Il y avait donc "des milliards de Francs Pacifique là-dessous ", selon les mots des frères Tetua

4. L'atoll de Kiritimati (Kiribati) présente lui aussi une vaste surface réticulée, mais qui ne couvre pas entièrement sa surface.

5. Le phosphate est surtout utilisé pour enrichir les engrais utilisés dans les grandes productions agricoles de par le monde.

6. La fiente d'oiseaux marins, le guano, est très riche en phosphate. Elle s'accumule avec le temps et peut constituer sur les îles récifales des gisements de quelques mètres à plusieurs dizaines de mètres d'épaisseur.

7. À Mataiva, l'origine du phosphate serait plutôt liée à des phénomènes de sédimentation de matières organiques sur au moins plusieurs millénaires (Rougerie et Jehl, 1994 ; Jehl, 1995; Jehl et Rougerie, 1995).

8. Nous n'avons pas réussi à recueillir des informations complémentaires sur ce point, en particulier quant au nom des sociétés impliquées (nationalités informées par les personnes interviewées ; voir également : https://www.tahiti-infos.com/ Extraction-de-phosphate-l-avenir-de-Makatea-entre-les-mains-de-la-population_a162995.html). Il semblerait également que le BRGM ait été impliqué, son rapport annuel 1981 faisant état de l'ouverture " d'un chantier pilote pour l'extraction du minerai de phosphate de l'atoll de Mataiva... afin de tester une drague expérimentale et de vérifier la validité d'une méthode originale d'extraction du minerai de phosphate " (BRGM, 1981:26). 
(entretiens du 08.02.2015 avec Laroche sur l'atoll voisin de Tikehau et du 13.02.2015 avec Edgar à Mataiva), Laroche ayant été maire de Mataiva entre 1996 et 2004 et Edgar étant le maire en 2015. En 2008, le maire de l'époque, Michel Cattiaux, évoquait la somme de 300 milliards de Francs Pacifique (http://consultation.ladepeche. pf/article/economie/phosphates-le-tavana-et-lapopulation-font-barrage) soit près de 2,5 milliards d'euros.

Les entreprises ont dû partager ces résultats avec les autorités du Territoire (gouvernement de Polynésie française), lesquelles ont donc voulu lancer l'exploitation sur 15 à 20 ans. Des réunions publiques ont dès lors été organisées avec la population pour expliquer le projet et les bénéfices que chacun pourrait en tirer. Ce ne sont pourtant pas les considérations économiques qui ont été les plus puissants leviers de l'opposition de la population à ce projet pharaonique. Trois autres arguments ont été mis en avant, en particulier par les anciens. D'abord, l'attachement profond des hommes à l'atoll en tant qu'être vivant. Dans la bouche des anciens,

«accepter l'exploitation du lagon, c'est sacrifier l'île. On a eu l'exemple à Makatea, et maintenant il n'y a que des trous là-bas. L'atoll est nourricier, c'est comme une maman. Il ne faut pas l'abîmer parce que sinon, on ne pourra plus vivre à Mataiva. " (Entretien du 14.02.2015 avec la présidente de l'association de Mataiva contre le phosphate)

Un deuxième argument s'appuyait sur l'expérience vécue par les habitants lors de la phase d'analyse de la qualité du gisement, dans la seconde moitié des années 1980 :

"au bout d'à peine quelques mois où ils avaient commencé à creuser dans le lagon pour tester le phosphate, on n'a plus pu manger les poissons du lagon, ils nous rendaient malade. Leurs tests n'ont duré que un an et demi, mais nous, on n'a pas pu manger le poisson du lagon pendant dix ans! Or, à Mataiva, on ne peut pas vivre sans manger le poisson du lagon, il est très important pour nous. » (Entretien du 12.02.2015 avec une femme âgée du village de Pahua)

Enfin, troisième argument, la peur de voir les zones d'exploitation s'étendre aux terres émergées. Le raisonnement était simple :

«s'il y a du phosphate dans le lagon, alors il y en a certainement sous les motus, et donc une fois qu'ils auront exploité le lagon, ils exploiteront nos terres. »

Ce point a soulevé beaucoup de discussions lors des réunions publiques, et en dehors, comme me l'ont rapporté Edgar Tetua et la présidente de l'association de Mataiva contre le phosphate (en- tretien du 14.02.2015). En effet, le phosphate n'est disponible qu'en-deçà de $5 \mathrm{~m}$ d'épaisseur de sol, et comme l'a exprimé le Territoire lors des rencontres avec les habitants, ces derniers ne sont propriétaires de leurs parcelles que jusqu'à $5 \mathrm{~m}$ de profondeur. En-deçà, l'espace est propriété du Territoire. Une telle situation ouvrait bien entendu la porte à des autorisations d'exploitation à terre, moyennant des pratiques d'expropriation plus ou moins douteuses. C'est ce dont les habitants ont eu peur, la propriété foncière étant dans les Tuamotu, comme partout ailleurs dans le Pacifique, le socle fondamental de la richesse familiale.

Le projet a fini par être abandonné, et ce n'est qu'en 2008 que de nouveaux investisseurs, Indiens ceux-là (Société Getax Coromandel), se sont manifestés. Mais là encore, sous l'impulsion des anciens et de la génération des 50-60 ans, la population s'est opposée à la proposition indienne (voir Phosphates: le Tavana et la population font barrage: http://consultation.ladepeche. pf/article/economie/phosphates-le-tavana-etla-population-font-barrage). L'argumentation restait la même, notamment la peur qu'on leur prenne leurs terres. Puis la succession des gouvernements ces dernières années a mis de nouveau ce projet de côté. Il n'en restait pas moins dans les tiroirs, puisqu'un rapport du Conseil Économique, Social et Culturel de Polynésie française et daté de janvier 2015 recommande

«l'éventuelle reprise de l'exploitation des gisements de Makatea et de Mataiva. " (Galenon et Sage, 2015 : 66)

À n'en pas douter, de nouveaux investisseurs se feront connaitre à plus ou moins court terme. Localement, cependant, la situation aura quelque peu évolué. En effet, les plus jeunes générations vont désormais faire leurs études en dehors de Mataiva - le collège à Rangiroa, puis le lycée à Tahiti - et comme dans bien des zones rurales du monde, l'attrait de la ville et de la modernité les fait se détourner assez brutalement des activités traditionnelles telles que le coprah et la pêche. Inéluctablement ${ }^{9}$, c'est le rapport à la terre nourricière qui s'érode, et avec lui la force de conviction des trois arguments précédents. La peur des anciens et des adultes de Mataiva, aujourd'hui, c'est de voir, demain, les jeunes générations céder.

\section{Les messages d'un atoll au monde}

Cette histoire d'un atoll en plein Pacifique n'est pas anecdotique. Elle rappelle par exemple celle d'un autre atoll, celui d'Aldabra (Seychelles, océan Indien), pour lequel le scientifique britannique David Ross Stoddart s'est battu, dès

9. À noter cependant que toutes les personnes interrogées ici avaient plus d'une trentaine d'années, et donc le point de vue des plus jeunes n'a pas été considéré. 
la fin des années 1960, pour qu'il soit classé au titre de Patrimoine mondial de l'unesco (Stoddart, 1968). D.R. Stoddart s'est engagé dans ce combat suite au projet d'installation d'une base militaire britannique qui aurait, pour des raisons logistiques évidentes (piste d'atterrissage, multiples bâtiments, creusement du récif pour créer des chenaux de navigation, etc.), irréversiblement bouleversé l'ordre écologique de cet atoll à la diversité biologique extraordinaire (Stoddart, 1967). L'atoll d'Aldabra a été classé au Patrimoine Mondial de l'unesco en 1982, et la Royal Air Force ne s'y est jamais implantée. Le combat du scientifique britannique aura duré près d'une quinzaine d'années, montrant qu'il s'agit toujours d'une bataille de longue haleine et de conviction. À Mataiva, cette bataille a débuté il y a près de 25 ans. Il serait nécessaire, de mon point de vue, de classer cet atoll au titre du patrimoine (géologique) de l'Humanité, du fait de son exceptionnel lagon réticulé et sur la base de la reconnaissance récente des " géosites " (site d'importance géologique). Si l'acte de labellisation (internationale ou nationale) n'est pas une panacée en soi, notamment parce qu'il s'accompagne souvent du développement d'un tourisme pas toujours soucieux de l'environnement, ou parce qu'il nécessite pour être efficace la mise en place de systèmes de gestion patrimoniale qui font parfois défaut dans certains contextes politiques et socioéconomiques, il aurait cependant le mérite, à Mataiva, de définitivement mettre un terme à la question de l'exploitation du phosphate du lagon.

Au-delà des atolls, l'histoire de Mataiva et de son phosphate, peut-être plus encore que celle d'Aldabra - puisque Mataiva est habité, ce qui n'était pas et n'est toujours pas le cas d'Aldabra -, est révélatrice des errances du monde moderne qui voit le combat s'intensifier entre des logiques de préservation de l'environnement et de développement local, d'une part, et de prédation des ressources à l'échelle globale, d'autre part. Ce qui est à préserver à Mataiva, comme dans bien d'autres endroits de la planète, c'est un patrimoine géologique, mais aussi un patrimoine culturel, identitaire, celui du rapport que les sociétés humaines ont pu construire avec l'environnement naturel, et que certaines d'entre elles entretiennent encore. Ainsi, Mataiva symbolise à sa façon le combat entre, d'une part, un développement fondé sur les ressources locales et les contraintes/limites qu'elles imposent et, d'autre part, un développement tiré par les logiques de la mondialisation et qui cherche à nourrir des populations avec les ressources d'autres populations. Cette seconde forme de développement, " hors-sol ", a d'abord pour effet pervers de générer des dégradations irréversibles des milieux et des écosystèmes, ce qui est, de l'avis de tous les spécialistes, de moins en moins tenable dans un contexte de grands bouleversements environnementaux tels que nous en connaissons aujourd'hui (changement climatique, acidification des océans, érosion massive de biodiversité, etc. - Gattuso et al., 2015). Ensuite, elle détruit également des systèmes de vie traditionnels, du moins ceux qui se sont adaptés aux contraintes imposées par l'environnement local. Sans parler de la déconnexion à l'environnement que cela génère au sein des populations qui, à l'autre bout du monde, voient arriver sur leurs marchés des biens de consommation, alimentaires ou autres, qu'elles ne lient pas intuitivement à l'exploitation en masse de ressources par nature limitées - puisque cette exploitation ne se fait pas « sous leurs yeux ». Par effet d'enchaînement, cette perte de conscience du lien consommation-ressources au sein des sociétés modernisées, puisqu'elle affranchit le consommateur de la contrainte " disponibilité des ressources ", libère en fait la consommation, qui grimpe en flèche et conduit, évidemment, à davantage d'exploitation (jusqu'à la surexploitation) des ressources naturelles. C'est une logique puissante dans le monde actuel, mais qui génère trop de paradoxes et pousse trop aux limites de la planète pour être tenable. Il y a donc un besoin crucial, pour ne pas dire désormais vital, de repenser cette relation développement/environnement, c'est-à-dire le rapport que les sociétés entretiennent avec les ressources naturelles, voire plus généralement avec «la Nature ». Au-delà de l'injonction, la question du comment faire s'impose.

De mon point de vue, cette réflexion d'ordre philosophique, mais finalement aussi d'ordre politique, économique et socioculturel, ne dépassera pas les sphères intellectuelles tant qu'elle ne sortira pas du domaine de l'abstrait. Elle doit prendre forme, concrètement. Or, des exemples concrets comme ceux de Mataiva ou d'Aldabra peuvent fournir matière à réflexion. La planète regorgeant malheureusement d'exemples de ce type, dans des milieux très divers les uns les autres, impliquant des thématiques elles-mêmes très variées, il existe inévitablement assez de cas différents pour capter l'attention du plus grand nombre. Il faut partir des exemples de terrain pour construire une force de conviction et de mobilisation. Ce n'est pas qu'une question d'écologie ; c'est une question de soutenabilité du développement humain. Comme quoi, des petits atolls isolés peuvent livrer de grands enseignements...

\section{Remerciements}

L'auteur remercie l'Agence nationale de la recherche scientifique (ANR) pour son soutien au projet CapAdapt, et le ministère de l'Écologie, 
du Développement durable et de l'Énergie pour son soutien au projet Réomers. Il remercie également Laroche et Edgar Tetua, pour lui avoir fait partager leur expérience de Mataiva et l'avoir accueilli avec chaleur et disponibilité. Il remercie également Virginie Duvat, Julien Rochette et l'un des relecteurs anonymes pour leurs précieux commentaires.

\section{BIBLIOGRAPHIE}

Assemblée de la Polynésie Française, 2017. Commission de l'économie, des finances, du budget et de la function publique, rapport $\mathrm{n}^{\circ} 87-2017,4 \mathrm{p}$.

BRGM, 1981. Rapport annuel 1981, Orléans, 61 p.

Decoudras Pierre-Marie, Danièle Laplace et Frédéric Tesson, 2005. Makatea, atoll oublié des Tuamotu (Polynésie française) : de la friche industrielle au développement local par le tourisme, Les Cahiers d'Outre-Mer 230, pp. 189-214.

Delesalle Bruno, J. Bell, Françoise BourrouilhLE JAN et al., 1985. Environmental survey of Mataiva atoll, Tuamotu archipelago, French Polynesia, Atoll Research Bulletin 286, 42 p.

Dupont Jean-François, Jacques Bonvallot et Jacques Florence, 1989. Un combat inégal : l'environnement des îles à phosphate du Pacifique face à l'industrie extractive, in Environnement, études de cas. Programme Régional Océanien de l'Environnement, Pacifique Sud Étude 4.

Duvat Virginie et Alexandre Magnan, 2012. Ces îles qui pourraient disparaître, Paris, Le PommierBelin.

Fiollet Luc, 2010. Nauru, lîle dévastée. Comment la civilisation capitaliste a détruit le pays le plus riche du monde, Paris, La Découverte.

Galenon Patrick et Winiki SAge (Rapporteurs), 2015 (21 janvier). L'avenir de la Polynésie française face à une gouvernance durable de son patrimoine marin, rapport adopté en assemblée plénière du Conseil économique, social et culturel de Polynésie française, 152/CEsC.

Gattuso Jean-Pierre, Alexandre Magnan, Raphaël BILlé et al., 2015. Contrasting Futures for Ocean and Society from Different Anthro- pogenic $\mathrm{CO}^{2}$ Emissions Scenarios, Science 349 (6243).

JeHL Caroline, 1995. Les mattes algaires cyanobactériennes (kopara) des atolls des Tuamotu: biochinie, productivité et signification écologique. Insertion dans un modèle de phosphatogénèse, doctorat de l'Université française du Pacifique, $332 \mathrm{p}$.

Jehl Caroline et Francis Rougerie, 1995. Phosphatogénèse en atolls polynésiens : la filiation mattes cyanobactériennes - phosphorites, Oceanologica Acta 18 (1), pp. 79-93.

LaCour Pierre, 1986 (séance du 17 novembre). Avis du Sénat, tome XXIII, Territoires d'outremer, annexe au procès-verbal, Commission des Affaires économiques et du plan, Assemblée nationale, Paris.

Magnan Alexandre et Virginie Duvat, 2015. Phosphate mining risks atoll culture, Nature 522 (https://doi.org/10.1038/522156b).

Pollock Nancy J., 2014. Nauru phosphate history and the resource curse narrative, Journal de la Société des Océanistes 138-139, pp. 107-119 (https://journals.openedition.org/jso/7055).

Rossfelder A.M., 1990. The submerged phosphate deposit of Mataiva atoll, French Polynesia, in W.C. Burnett \& S.R. Riggs (éds.), Phosphate Deposits of the World: Neogene to modern phosphorites, Cambridge, Cambridge University Press, pp. 195-204.

Rougerie Francis et Caroline Jehl, 1994. Le dossier Phosphate revisité : controverses et nouveau modèle, in Actes des troisièmes journées de la recherche en Polynésie française (27-28 octobre 1993), pp. 48-62.

Rougerie Francis, 1995. Nature et fonctionnement des atolls des Tuamotu (Polynésie française), Oceanologica Acta 18 (1), pp. 61-78.

SodTER François, 1985. Eléments d'une histoire démographique, in Contribution à l'etude de l'atoll de Tikehau (archipel des Tuamotu, Polynésie française), orstom Tahiti, Notes et Documents Océanographiques 24, pp. 125-134.

StODDART David Ross, 1967. Ecology of Aldabra atoll, Nature 25, pp. 1174-1177.

-, 1968. The Aldabra affair, Biological Conservation 1 (1), pp. 63-69. 